\title{
ANÁLISE DO DESEMPENHO DO SALTO VERTICAL DE MILITARES SUBMETIDOS A DIFERENTES MÉTODOS DE TREINAMENTO DE FLEXIBILIDADE
}

\section{Analysis of vertical jump performance of military subjects submitted to different methods of stretching training}

\author{
Carlos José Nogueira ${ }^{1,3}$, Rodrigo Gomes de Souza Vale ${ }^{3}$, Tânia dos Santos Giani ${ }^{3}$, Estélio Henrique Martin Dantas ${ }^{1,3}$ \\ ${ }^{1}$ Universidade Castelo Branco - UCB, Rio de Janeiro - RJ - Brasil. \\ ${ }^{2}$ Faculdade Estácio de Sá, Juiz de Fora - MG - Brasil. \\ ${ }^{3}$ Laboratório de Biociências da Motricidade Humana - LABIMH - Rio de Janeiro - RJ - Brasil.
}

\begin{abstract}
Resumo: Exercícios de flexibilidade são frequentemente aplicados anteriormente às atividades físicas e competitivas. No entanto, não está claro na literatura se a flexibilidade pré-exercício traz benefícios ou prejuízos para o desempenho. Assim, o objetivo desse estudo foi comparar os efeitos agudos do alongamento estático submáximo e do método de facilitação neuromuscular proprioceptiva (FNP) sobre o desempenho no salto vertical. Vinte militares do gênero masculino realizaram três saltos verticais, antes, imediatamente após e em 10, 20, 40 e 60 minutos após as seguintes condições: a) Grupo Controle (GC); b) Alongamento Estático Submáximo (GA); c) FNP (GFNP). Os testes foram realizados em duas Plataformas de Contato (Multisprint / Hidrofit), em dias separados, aleatoriamente, e com intervalo mínimo de 48 horas entre as sessões. A ANOVA multivariada de medidas repetidas, seguida do post hoc de Tukey, revelou uma redução significativa no desempenho do salto vertical do GFNP pré em comparação com GFNP pós (11,1\%), com redução significativa por até 10 minutos (9,9\%), observada na comparação do GFNP S10 em relação à GFNP pré. Ao contrário, os valores intragrupos para o GC e GA não apresentaram diminuições significativas na comparação pré e pós-teste e entre S10, S20, S40 e S60 em relação ao GC pós. Na comparação intergrupos, houve diferença significativa entre GC pós vs GFNP pós $(p=0,012)$ e entre GFNP pós vs GA pós $(p=0,038)$. Conclui-se que o desempenho do salto vertical com contramovimento é reduzido após a aplicação do método de FNP em comparação ao alongamento estático submáximo e a condição de controle.
\end{abstract}

Palavras-chave: Flexibilidade, facilitação neuromuscular proprioceptiva, salto vertical.

\begin{abstract}
Flexibility exercises are frequently used before physical activity and competitions. However, it is not clear in the literature whether pre-exercise stretching is helpful or harmful to performance. The aim of this study was to compare the acute effects of sub-maximal stretching and proprioceptive neuromuscular facilitation (FNP) method stretching on performance in the vertical jump. Twenty male members of the military made three vertical jumps, before, immediately after, and 10, 20, 40 and 60 minutes after the following conditions: a) Control Group (GC); b) Sub-maximal Static Stretching (GA); c) FNP (GFNP). The tests were performed on two Contact Platforms (Multisprint / Hidrofit), on separate days, randomly, and with a minimum interval of 48 hours between sessions. Multivariate repeated measures ANOVA, followed by the Tukey post hoc test, showed a significant reduction in the performance in the vertical jump of the GFNP pre in comparison with GFNP posts (11,1\%), with a significant reduction for up to 10 minutes $(9,9 \%)$ noted in comparison between the GFNP S10 and the GFNP pre. To the contrary, the intra-group values for the GC and GA did not record significant reductions in the comparison pre and post test between S10, S20, S40 and S60 compared to the GC post. In the intergroup comparisons, there was a significant difference between GC post and GFNP post $(p=0,012)$ and between GFNP post vs. GA post $(p=0,038)$. We conclude that the performance of the vertical jump with countermovement is reduced after the use of the FNP method, in comparison with sub-maximal stretching and the control condition.
\end{abstract}

Keywords: Flexibility, proprioceptive neuromuscular facilitation, vertical jump.

Aceito em 27/11/2009 - Rev. Educ. Fís. 2009 - 03-8. Rio de Janeiro - RJ - Brasil

\section{INTRODUÇÃO}

O treino de flexibilidade é utilizado com bastante frequência nos contextos clínico e desportivo(1). Os principais métodos de treinamento da flexibilidade são o alongamento estático, o dinâmico, o balístico e a facilitação neuromuscular proprioceptiva (FNP) ${ }^{(2)}$. Aeficiência dessas técnicas está diretamente relacionada a forma com que elas são utilizadas ${ }^{(3)}$. Fatores como frequência, duração, e, principalmente a intensidade ${ }^{(4,5)}$, podem influenciar a flexibilidade, 
gerando controvérsias com relação a sua forma de aplicação e quanto às conseqüências sobre o desempenho do indivíduo(6).

Os exercícios de flexibilidade, aplicados anteriormente às atividades físicas e competitivas, têm apresentado diferentes resultados no desempenho do indivíduo(6). Os métodos de alongamento estático e FNP, utilizados como aquecimento pré-exercício, tem mostrado efeitos negativos no pico de torque ${ }^{(7,8)}$, na força máxima, dinâmica e potência( ${ }^{(9,10,11,12)}$.

O alongamento estático submáximo como pré-exercício, aplicado até antes do desconforto muscular, não tem demonstrado mudanças nos valores do salto vertical(13,14,6). Todavia, estudos envolvendo o alongamento estático máximo (além do ponto de desconforto muscular)(15) e o método de $\operatorname{FNP}(16,15,17)$, apresentaram reduções nos valores do salto vertical, comprometendo o desempenho para essa atividade.

Ainda são escassos os estudos que comparam os efeitos combinados desses métodos sobre a potência e força explosiva no salto vertical. Não obstante, as pesquisas que tratam dos efeitos do alongamento estático e FNP sobre o desempenho muscular produzem resultados conflitantes (15). As controvérsias existentes não permitem uma definição clara de qual o melhor método de flexibilidade para ser utilizado como préexercício. Isso dificulta a prescrição e orientação dessa qualidade em treinamentos e programas de atividade física, e sua utilização como aquecimento antes de exercícios e competições. Portanto, o objetivo do presente estudo foi comparar os efeitos agudos do alongamento estático submáximo e do método de FNP sobre o desempenho no salto vertical. O presente estudo apresenta como hipótese que a aplicação do método de FNP afetará negativamente a performance dos sujeitos em comparação com o alongamento estático submáximo.

\section{METODOLOGIA}

\section{Amostra}

A amostra, selecionada de forma intencional e por conveniência, foi composta por 20 militares adultos, do sexo masculino (média e desvio padrão; idade: 20,05 $\pm 1,79$ anos; estatura: $173 \pm 0,07$ cm; peso: $64,33 \pm 5,13 \mathrm{~kg}$ ). Todos os voluntários praticavam atividade física regularmente, no mínimo três vezes por semana e duas horas por dia, há pelo menos um ano.

Antes da coleta de dados, os voluntários foram informados a respeito dos objetivos e dos procedimentos do respectivo estudo e orientados a evitar atividade física intensa por 24 horas antes das sessões de treino. Todos os sujeitos responderam negativamente aos itens do questionário de prontidão para a prática de atividade física (PAR-Q) e assinaram o termo de consentimento livre e esclarecido conforme resolução 196/96 para experimentos com humanos e a declaração de Helsinki ${ }^{(18)}$. O presente estudo foi aprovado pelo Comitê de Ética e Pesquisas em Seres Humanos da Universidade Castelo Branco - RJ (protocolo 0005/2008 expedido em 19/02/2008).

\section{Procedimentos}

Os procedimentos para a realização do teste de salto vertical e aplicação das rotinas de flexibilidade foram desenvolvidos, de forma aleatória, em dias separados, com intervalo mínimo de 48 horas entre as sessões, nas condições a seguir: (a) sem aplicação de flexibilidade (GC - grupo controle); (b) com a aplicação do alongamento estático submáximo (GA); (c) com a aplicação do método de FNP (GFNP). Foi realizada uma sessão preliminar na qual cada voluntário realizou três tentativas do teste de salto vertical para familiarização com o mesmo. Como procedimento geral os sujeitos realizaram três saltos verticais com contramovimento antes, e, imediatamente após cada condição, sendo registrado o melhor salto entre as tentativas executadas, e um aquecimento submáximo de 10 minutos (60 a $70 \%$ da freqüência cardíaca máxima) em um cicloergômetro (Movement, Brasil).

Para a realização do salto vertical foram utilizadas duas plataformas (placas de contato) e o software Multisprint 1.20, pertencentes ao Kit Multsprint / Hidrofit (Brasil)(17) . Utilizou-se a técnica do salto com contramovimento - na qual o indivíduo realiza um salto com movimento de preparação (contramovimento), sem a ajuda dos braços. As placas de contato registram os tempos de vôo, sendo que, a partir desse parâmetro, é calculada a altura do salto(19).

$\mathrm{Na}$ condição de GC, os voluntários realizaram 
o teste de salto vertical sem flexibilidade. Após 10 minutos (tempo correspondente à duração das rotinas de flexibilidade), os voluntários realizaram novo teste (S-pós). Na seqüência, os voluntários realizaram saltos em 10, 20, 40 e 60 minutos após S-pós. Para a condição de GA, os sujeitos realizaram o salto vertical e, logo após, foi aplicada uma rotina de alongamento estático submáximo com três séries de seis segundos de sustentação estática, dentro dos limites normais de amplitude de movimento (ADM) sem desconforto muscular - nível 31a 60 (51,5 \pm 0.47) da escala PerFlex ${ }^{(21)}$, com intervalo de cinco segundos, semelhante ao utilizado por César et al.(20). Imediatamente após, realizou-se o teste de salto vertical (SAlong-pós) e 10, 20, 40 e 60 minutos pós-alongamento. $\mathrm{Na}$ condição deFNP, após o teste, os sujeitos realizaram uma rotina de FNP, na qual o grupamento muscular foi contraído isometricamente durante cinco segundos e sustentado estaticamente durante 30 segundos na posição do movimento, além do ponto de desconforto - nível 61 a $80(70.1 \pm 0.45)$ da escala PerFlex (21), em quatro séries com intervalo de 20 segundos entre as mesmas ${ }^{(11)}$. O salto vertical foi realizado após a sessão de FNP e em 10, 20, 40 e 60 minutos pós-FNP. Os movimentos utilizados nas sessões de alongamento estático e FNP são os envolvidos diretamente na técnica do salto vertical: flexão do quadril (FQ) com o joelho estendido em decúbito dorsal, flexão dorsal do tornozelo (FD) com o indivíduo em decúbito dorsal, flexão do joelho (FJ) com o indivíduo em decúbito ventral(17).

Para avaliar a percepção do esforço nas sessões de Alongamento e FNP, utilizou-se um método adimensional, denominado Escala de Esforço Percebido na Flexibilidade (PERFLEX)(21), que possui cinco níveis de intensidades, variando de 0 a 110, categorizados em cinco descritores verbais, para que o avaliando possa discernir, através da sua percepção, qual a sensação correspondente à amplitude de movimento realizado.

\section{Análise Estatística}

Os dados foram apresentados como média e desvio padrão. Utilizou-se o teste de ShapiroWilk para verificar a normalidade dos dados e a homogeneidade das variâncias foi analisada pelo teste de Levene. A análise de variância (ANOVA) multivariada de medidas repetidas foi empregada para as comparações intra e intergrupos, seguida do post hoc de Tukey para identificar as possíveis diferenças. Utilizou-se o programa SPSS (14.0 for Windows) para a realização do tratamento dos dados adotando-se o valor de $p<0,05$ para a significância estatística.

\section{RESULTADOS}

Na FIGURA 1 são apresentados os resultados da comparação intra e intergrupos, apresentados em média e desvio padrão, para os grupos controle (GC), GA e GFNP obtidas entre as condições pré e pós, e, 10, 20, 40 e 60 minutos pós.

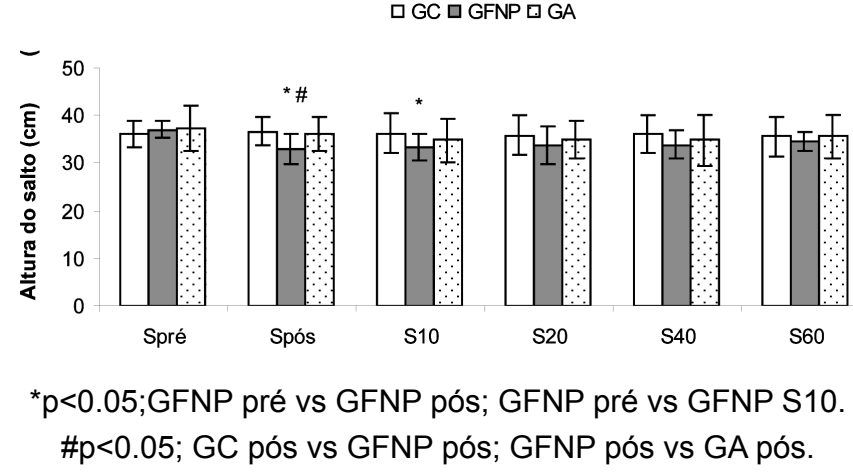

Ao analisar a FIGURA 1, nota-se que houve redução significativa no desempenho do salto vertical do GFNP pré em comparação com GFNP pós $(11,1 \%)$, com redução significativa por até 10 minutos $(9,9 \%)$, observada na comparação do GFNP $\mathrm{S} 10$ em relação à GFNP pré. Ao contrário, os valores intragrupos para o GC e GA não apresentaram diminuições significativas na comparação pré e pós-teste e entre S10, S20, S40 e S60 em relação ao GC pós. Na comparação intergrupos, houve diferença significativa entre GC pós vs GFNP pós ( $p=0,012$ ) e entre GFNP pós vs GA pós $(p=0,038)$.

\section{DISCUSSÃO}

Os resultados do presente estudo revelaram uma diminuição significativa nos valores do salto vertical após a aplicação do método de FNP em comparação com o alongamento e grupo controle, suportando a hipótese de comprometimento no desempenho dos saltos após a utilização do FNP. Os resultados corroboram os achados de 
Nogueira et al.(17), que verificaram o efeito agudo do alongamento estático e do método de FNP em 12 adultos do sexo masculino e encontraram uma redução significativa na força explosiva após a aplicação do FNP, mas não obtiveram modificações significativas no grupo que foi submetido ao alongamento. A semelhança com o presente estudo refere-se à utilização do mesmo protocolo de aplicação da flexibilidade, contudo, não foram realizados saltos por um período de até 60 minutos pós-flexibilidade, limitando à pesquisa quanto a uma possível investigação sobre a duração dos efeitos agudos do alongamento e do FNP sobre a força explosiva.

Ao verificarem se diferentes rotinas de flexibilidade utilizadas como aquecimento afetariam o salto vertical, Church et al.(16) apresentaram resultados bastantes semelhantes aos do presente estudo, encontrando uma redução significativa do salto $(p=0,01)$ após a aplicação do FNP. Já Young e Elliot(22) encontraram uma redução de $6,91 \%(p=0,026)$ no desempenho do salto com queda após o alongamento estático e FNP.

Bradley et al.(15) observaram uma redução significativa no salto vertical após o alongamento estático máximo $(4,0 \%)$ e FNP $(5,1 \%)$ em 18 universitários do sexo masculino, concordando com os resultados do presente estudo quanto à aplicação do método de FNP e discordando quanto ao alongamento. Cornwell et al.(23) investigaram os efeitos do alongamento estático máximo sobre o desempenho no exercício de agachamento e salto com contramovimento (CMJ) em 10 homens adultos e verificaram uma diminuição significativa na performance do CMJ com a aplicação de três séries com 30 segundos de sustentação máxima. Uma das razões que podem explicar a redução do salto pós-alongamento nos estudos acima se refere à intensidade dos protocolo utilizados, ao contrário do alongamento submáximo utilizado na presente pesquisa.

Não houve mudanças significativas na altura do salto vertical $(p=0,66>0,05)$ entre os testes pré e pós alongamento estático e FNP em nove tenistas adolescentes ${ }^{(4)}$, assim como no estudo de Unick et al.(6), no qual não houve diferenças significativas nos valores do salto vertical como resultado do alongamento estático e balístico em 16 basquetebolistas do sexo feminino. Ambos os estudos utilizaram protocolos semelhantes para o alongamento estático, envolvendo três séries com 15 segundos de sustentação, abaixo do ponto de desconforto muscular, sendo a intensidade submáxima uma possível causa da não ocorrência de mudanças no salto pós-flexibilidade. Os autores de ambos os estudos concluíram que, atletas que necessitam diretamente da força muscular para geração de potência, parecem não ser beneficiados com exercícios de alongamento estático submáximo nem FNP antes da prática específica.

No presente estudo houve redução significativa no desempenho por um período de até 10 minutos após a aplicação do método de FNP. Fowles et al.(24), ao avaliarem a força de contração máxima dos flexores plantares após uma sessão de alongamento passivo, encontraram uma redução significativa de $28 \%$, a qual persistiu por até 60 minutos após o término do alongamento. Além da intensidade, fatores como o tipo de força e o grupamento muscular avaliados, podem ser os responsáveis pelo acentuado tempo de permanência dos efeitos do alongamento sobre a performance.

Alterações da rigidez muscular(11,6) e fatores neurais ${ }^{(24,22)}$ são atribuídos como possíveis causas do déficit de força induzido pelo alongamento. Portanto, constitui uma limitação do presente estudo o fato de não serem investigados os mecanismos responsáveis pelo decréscimo nos valores do salto vertical após o método de FNP.

\section{CONCLUSÃO}

Com base nos resultados encontrados, conclui-se que o desempenho do salto vertical com contramovimento foi reduzido após a aplicação do método de FNP em comparação ao alongamento estático submáximo e a condição de controle. Portanto, essa técnica de flexibilidade seria contra indicada como pré-exercício ou aquecimento para atividades que envolvem a execução do salto vertical, ao contrário do alongamento estático submáximo que parece não influenciar negativamente o desempenho. As evidências levantadas nesse estudo poderão servir de base para uma adequada prescrição de exercícios, por parte dos profissionais que atuam na área do treinamento e reabilitação, quanto à indicação da 
técnica de FNP antes das sessões de treino, como aquecimento, ou em protocolos de reabilitação. Futuros estudos devem considerar, além dos efeitos agudos em diferentes populações com diferentes intensidades de aplicação, a análise dos mecanismos responsáveis pelos efeitos negativos do método de FNP sobre a performance do salto vertical.

\section{REFERÊNCIAS BIBLIOGRÁFICAS}

1. Coelho LFS. O treino da flexibilidade muscular e o aumento da amplitude de movimento: uma revisão de crítica da literatura. Revista do Desporto e Saúde 2007; 4(4): 59-70.

2. Gama ZAS, Medeiros CAS, Dantas AVR, Souza TO. Influência da freqüência de alongamento utilizando facilitação neuromuscular proprioceptiva na flexibilidade dos músculos isquiotibiais. Revista Brasileira de Medicina do Esporte 2007; 13(1): 3338.

3. Rosa AC, Montandon I. Efeitos do aquecimento sobre a amplitude de movimento: uma revisão crítica. Revista Brasileira de Ciência e Movimento 2006; 14(1): 109-116.

4. Carvalho FLP, Prati JELR, Carvalho MCGA, Dantas EHM. Acute effects of static stretching and proprioceptive neuromuscular facilitation on the performance of vertical jump in adolescent tennis players. Fitness Performance Journal 2009; 8(4): 264-268.

5. Chagas $\mathrm{MH}$, Bhering EL, Bergamini JC, Menzel HJ. Comparação de duas diferentes intensidades de aplicação de alongamento na amplitude de movimento. Revista Brasileira de Medicina do Esporte 2007;14(2):99-103.

6. Unick J, Kieffer HS, Cheesman W, Feeney A. The acute effects of static and ballistic stretching on vertical jump performance in trained women. Journal of Strength and Conditioning Research 2005; 19(1): $206-212$.

7. Brentano MA, Rodrigues LP, Kruel LFM. Efeitos de diferentes sessões de aquecimento no torque e amplitude articular de homens jovens. Revista Brasileira de Educação Física e Esportes 2008; 22(1):53-62.

8. Cramer JT, Housh TJ, Johnson GO, Miller JM, Coburn JW, Beck TW. Acute effects of static stretching on peak torque in women. Journal of Strength and Conditioning Research 2004; 18(2):236-241.
9. Bacurau RFP, Ugrinowitsch C,Tricolo V, Cabral LF, Aoki, MS. Acute effect of a ballistic and a static stretching exercise bout on flexibility and maximal strength. Journal of Strength and Conditioning Research 2009; 23(1):304-308.

10. Endlich PW, Farina GR, Dambroz C, Gonçalves WLS, Moysés MR, MILL JG, Abreu GR. Efeitos agudos do Alongamento Estático no desempenho da Força Dinâmica em homens jovens. Revista Brasileira de Medicina do Esporte 2009; 15(3):200203.

11. Marek SM, Cramer JT, Fincher AL, Massey LL, Dangelmaier SM, Purkayastha S, Fitz KA, Culbertson JY. Acute Effects of Static and Proprioceptive Neuromuscular Facilitation Stretching on Muscle Strength and Power Output. Journal of Athletic Training, Columbus 2005; 40(2): 94-103.

12. Yamaguchi T, Ishii K, Yamanaka M, Yasuda K. Acute effect of static stretching on power output during concentric dynamic constant external resistence leg extension. Journal of Strength Conditionning Research 2006; 20(4): 804-810.

13. Fantini C, Araújo S, Pacheco F, Menzel HJ. Acute effect of quadríceps stretching on vertical ground reaction force, muscle activity and vertical jump performance. XXIV ISBS Symposium 2006; Salzburg - Austria.

14. Power K, Behm D, Cahill F, Carroll M, Young W. An Acute Bout of Static Stretching: Efects on Force and Jumping Performance. Medicine \& Science in Sports \& Exercise 2004; 36(8): 1389-1396.

15. Bradley PS, Olsen PD, Portas MD. The effect of static, ballistic, and proprioceptive neuromuscular facilitation stretching on vertical jump performance. Journal of Strength and Conditioning Research 2007; 21(1):223-226.

16. Church JB, Wiggins MS, Moode EM, Crist R. Effect of warm-up and flexibility treatments on vertical jump performance. Journal Strength Conditioning Research 2001;15(3):332-336.

17. Nogueira CJ, Galdino LAS, Vale RGS, Dantas EHM. Efeito agudo do alongamento submáximo e do método de Facilitação Neuromuscular Proprioceptiva sobre a Força Explosiva. HU Revista 2009; 35(1): 43-48.

18. World Medical Association Declaration of Helsinki. Ethical Principles for Medical Research Involving Human Subjects. 59th WMA General Assembly 2008; Seoul. 
19. Galdino LAS, Nogueira CJ, César EP, Fortes MEP, Perrout JR, Dantas EHM. Comparação Entre Níveis de Força Explosiva de Membros Inferiores Antes e Após Flexionamento Passivo. Fitness \& Performance Journal 2005; 4(1):11-15.

20. César EP, Bara filho MG, Perrout JRL, Aidar FJ, Dantas EHM. Modificações agudas dos níveis séricos de creatina quinase em adultos jovens submetidos ao trabalho de flexionamento estático e de força máxima. Revista de Desporto e Saúde 2008; 4(3): 49-55.

21. Dantas EHM, Salomão PT, Vale RGS, Achour júnior A, Simão R, Figueiredo NMA. Escala de esforço percebido na flexibilidade (PERFLEX): um instrumento adimensional para se avaliar a intensidade? Fitness Performance Journal 2008; 7(5): 289-294.

22. Yamaguchi T, Ishii K, Yamanaka M, Yasuda K. Acute effect of static stretching on power output during concentric dynamic constant external resistence leg extension. Journal of Strength Conditionning Research 2006; 20(4): 804-810.
23. Cornwell A, Nelson AG, Sidaway B. Acute Effects of stretching on the neuromechanical properties of the triceps surae muscle complex. European Journal Appl. Physiol 2002; 86: 428-434.

24. Fowles JR, Sale G, Macdougall JD. Reduced strength after passive stretch of the human plantarflexors. Journal Applied Physiology 2000; 89:1179-1188.

Endereço para correspondência

Carlos José Nogueira, Rua Esperanto,185, São Miguel, Santos Dumont, Cep 36240-000, Minas Gerais, Brasil. carlosjn29@yahoo.com.br

Rodrigo Gomes de Souza Vale, Rua Oscar Clark, 805 Pq. Mataruna CEP: 28970-000 Araruama, RJ, Brasil.rodrigovale@globo.com

Estélio Henrique Martin Dantas, Rua Prof. Hermes Lima, 238 - Cob.02 - Recreio dos Bandeirantes - CEP: 22795-065 - Rio de Janeiro / RJ - Brasil. estelio@cobrase.org.br 